\title{
Mes Mille et Une Nuits
}

\section{Jean Martin}

Dr méd., membre de la rédaction

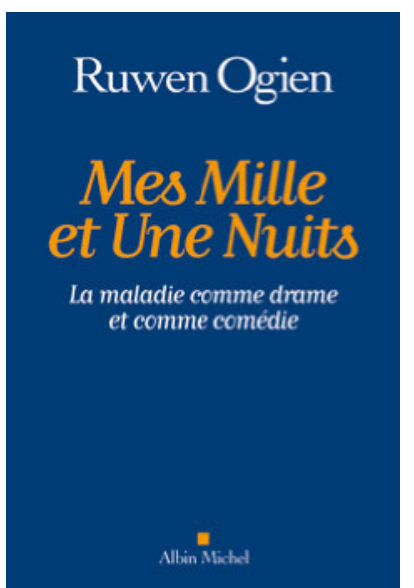

Ruwen Ogien

\section{Mes Mille et Une Nuits}

La maladie comme drame et comme comédie Paris: Albin Michel; 2017.

254 pages. $31.50 \mathrm{CHF}$.

ISBN 978-2-226-39524-5

Philosophe analytique français Ruwen Ogien, 70 ans, est notamment l'avocat d'une éthique minimaliste (en bref: nous ne devons rien à nous-mêmes, «ne pas nuire aux autres, rien de plus»). En 2013 a été diagnostiqué chez lui un cancer du pancréas; il a été opéré, a subi une demi-douzaine de chimiothérapies et vient de publier le livre discuté ici (qu'il a présenté à fin janvier 2017 dans une émission littéraire de la chaîne France 5). Je l'abordais avec des attentes mitigées: je ne suis pas un tenant de l'éthique minimaliste et imaginais trouver un journal de maladie comme d'autres, anecdotique. J'en sors en recommandant cet ouvrage à qui souhaite, à l'occasion d'une histoire individuelle, bénéficier aussi d'un tour d'horizon bien informé de sociologie médicale (les relations soigné-soignant, les rapports entre soignants) et de psychologie médicale (ressentis du malade, entre autres) - un commentateur parle de «méditation sur la maladie et la relation médecin-malade».

Beaucoup d'érudition dans Mes Mille et Une Nuits, y compris une bibliographie de 120 titres, sélection d'ou- vrages académiques d'Europe et d'Amérique du Nord et de classiques littéraires comme Mars, de notre compatriote Fritz Zorn, ou Une mort très douce, de Simone de Beauvoir. Souvent cités, à juste titre, l'Américain Talcott Parsons [1] et les Français Adam et Herzlich [2]. Vécu personnel. Mélange des sentiments: «Je suis atteint d'un cancer capricieux. Je crois être indifférent à ce qui m'arrive mais suis terriblement inquiet lorsque je dois aller chercher les résultats des analyses. Je me sens plein de compassion envers les autres malades mais ai du mal à supporter leur proximité physique. J'ai l'impression d'être de plus en plus étranger à mon corps alors que je m'intéresse sérieusement à son fonctionnement pour la première fois. Je prétends être ouvert à toutes les thérapies alternatives mais m'arrange pour n'en suivre aucune. J'éprouve de la gratitude et même de l'amour pour le personnel soignant mais ressens aussi souvent de la méfiance et de la crainte à son égard.»

Contre le dolorisme. Ogien s'en prend aux vues doloristes de la maladie et de la souffrance, mises en évidence par certains auteurs et milieux, religieux par exemple. A son sens, ces réponses "contribuent à discréditer la souffrance des personnes atteintes de graves maladies, à renforcer la violence sociale à leur encontre [liée à ce qu'on voit à la souffrance une vertu «rédemptrice»], et à protéger certaines formes de paternalisme médical.» Son intuition: "La souffrance physique est un fait brut qui n'a aucun sens, qu'on peut expliquer par des causes, mais qu'on ne peut pas justifier par des raisons» - ceci même si bien sûr (note de J.M.) personne n'entendrait s'opposer à la volonté de la personne qui ne souhaite pas qu'on calme ses douleurs.

\section{"La souffrance physique est un fait brut qui} n'a aucun sens, qu'on peut expliquer par des causes, mais qu'on ne peut pas justifier par des raisons.»

La maladie come métaphore (on pense au livre de Susan Sontag). L'auteur se penche sur la manière dont les écrits sur la maladie s'organisent autour de trois métaphores: 1) celle de l'épreuve ou du défi, «psychologique et encombrée de métaphysique existentielle»; 2) celle du «royaume» où on s'installe contre son gré, 
"sociale et dégagée de toute métaphysique»; 3) celle du métier: "être malade serait comme une profession exigeant un apprentissage, un savoir faire, une planification active». C'est cette dernière qu'il préfère.

Il discute longuement ce que depuis Talcott Parsons on appelle statut et rôles «standards» du malade. "J'avais l'impression que, si je ne voulais pas être perçu comme un 'déchet' ne méritant pas des efforts thérapeutiques, je devais présenter une certaine image: celle d'une personne résistant vaillamment, sincèrement désireuse de suivre les recommandations des médecins.» Vision traditionnelle, contre-balancée aujourd'hui par l'accent mis sur les droits des patients, qui ont fait dans nos pays l'objet de textes législatifs, avec en tête le thème de l'autonomie du malade - et l'émergence du patient-expert.

Rôle du milieu. Rappel de son importance: c'est là «où s'expriment les relations de la personne à son milieu social que se définit le sens même de l'expérience $\mathrm{du}$ malade et que s'orienteront ses comportements». Avec la référence au récit de Fritz Zorn [3], où pour le héros l'annonce de la maladie provoque un sentiment de libération à l'égard d'obligations sociales étouffantes. Ogien à ce propos: «Je n’ai aucune envie de m'apitoyer sur moi-même mais je ne ressens pas non plus de joie douce à la manière de Zorn.» Ce fait sociologique: "Pour les classes populaires la maladie est [dans une plus grande mesure] une menace matérielle, alors que, pour les classes moyennes et supérieures, elle est plutôt perçue comme un obstacle au bonheur personnel.»

Qualité de relation. "Rendez-vous avec V.M., mon médecin anti-douleurs. C'est un spécialiste des soins palliatifs, ce qui pourrait être inquiétant, mais il sait toujours trouver les mots non pas nécessairement pour me rassurer, mais pour me faire comprendre. Grâce à lui, je vois mieux la différence concrète entre s'occuper du malade et s'intéresser seulement à la maladie.»

Des réserves à l'endroit de notions admises. S'agissant des réactions du malade à l'annonce et au cours d'une affection grave, l'auteur dit ses réserves à l'endroit de la séquence décrite par Elisabeth Kübler-Ross, avec ses cinq stades: déni (denial), colère (anger), négociation (bargaining), dépression, acceptation. Sans doute ce schéma n'est-il pas toujours vérifié («jamais et toujours n'existent pas en médecine», disait un de nos maîtres), mais la contribution de Kübler-Ross reste à mon sens d'importance.

Réserves aussi vis-à-vis de la psychologie dite positive et d'un mot à la mode, la résilience. "Au fond, la psychologie positive, dont la résilience est un des piliers, a un côté bêtement optimiste, répugnant aux yeux de tous ceux dont la vie est précaire, marquée par des échecs et des peines profondes. Elle tend à culpabiliser tous les défaitistes en pensée, ceux qui n'ont pas la force ou l'envie de surmonter leur désespoir [...] C'est pourquoi la tendance à présenter les maladies comme des défis susceptibles de nous faire grandir m'est devenue quasiment insupportable.» Si résilience et autres notions positives méritent sans doute de retenir l'attention, il faut aussi entendre la crainte que ce discours puisse augmenter le malheur des 'paumés'.

Une idée iconoclaste. Les maladies chroniques prennent de plus en plus de place, tous s'en préoccupent. Beaucoup d'affections dont on ne guérit pas mais où l'objectif est la poursuite de l'existence avec la meilleure qualité de vie. Ogien emprunte à d'autres une crainte surprenante: "Le fait que les médicaments anticancéreux n'ont pour objectif que de prolonger la survie donne l'impression d'un grand déficit de l'insti-

\section{Si résilience et autres notions positives méri-} tent sans doute de retenir l'attention, il faut aussi entendre la crainte que ce discours puisse augmenter le malheur des 'paumés'.

tution médicale. Ceci devrait être un aiguillon pour revenir à un but plus classique, la guérison. Mais l'invasion des maladies chroniques fait tellement les affaires de l'industrie pharmaceutique qu'on peut se demander si cette dernière ne contribue pas à laisser les choses en l'état. Certains chercheurs font même l'hypothèse que c'est la relative impuissance des médicaments à guérir la maladie qui en font tout le prix pour l'industrie.» Well...? Noter que l'auteur ne reprend pas sans autre cette thèse à son compte.

Enfin: un point délicat mais pas éthiquement irrecevable. "Sans tomber dans un utilitarisme calculateur, je me demande, comme devrait le faire tout citoyen 'raisonnable', si prolonger ma vie de quelques semaines au prix de dépenses énormes en vaut vraiment la peine [...] Est-ce que je trouverais juste de dépenser tout ce qui me reste à la banque pour cela, en privant ma famille ou une organisation caritative de cette somme qu'ils pourraient consacrer à un bien-être plus durable. Je suis en train de perdre mes certitudes 'déontologiques' ma croyance que l'impératif de me maintenir en vie prévaut sur toutes les autres considérations.»

\section{Références}

1 Parsons T. Eléments pour une sociologie de l'action. Paris: Plon; 1955.

2 Adam Ph, Herzlich C. Sociologie de la maladie et de la médecine. Paris: Armand Colin; 1994

3 Zorn F. Mars. Paris: Gallimard; 1979. 